\title{
Immunological properties and biological effectiveness of insulin analogues substituted at position B30
}

\author{
H.P. Neubauer, R. Obermeier and G. Schnorr \\ Pharmaceutical Research Division, Hoechst AG, Frankfurt/Main, FRG
}

\begin{abstract}
Summary. The aim of this investigation was to assess the immunological properties of several insulin analogues by examining both their antigenic and immunogenic behaviour. In addition, the hypoglycaemic activity was also determined and compared with values obtained with natural insulins. The modified insulin preparations were of porcine and bovine origin. All analogues had in common the fact that the alanine B30 had been exchanged by either leucine, threonine, tyrosine, phenylalanine or glycine. The blood glucose-lowering activity was determined in rabbits and dogs, while the stimulation of antibody development was studied in three different animal species; pigs, dogs and rabbits. The antigenic properties of analogues were evaluated in vitro by measuring their
\end{abstract}

binding capacity to pre-formed antibodies. In all cases the blood glucose lowering activity of the analogues was comparable to that of the respective natural insulin. There were remarkable differences in the binding capacity to pre-formed antibodies, with bovine Leu-B30 insulin competing only to $73 \%$ with the natural insulin tracer. With regard to antibody development, the analogues behaved similarly to the original hormones. These results show that there is little correlation between the antigenic make-up of the insulin molecule and its ability to provoke antibody stimulation

Key words: Insulin analogues, immunogenicity, antigenicity, efficacy
Compared with its porcine counterpart, beef insulin is distinguished by a rather high degree of immunogenicity [1-3]. The A-chain loop is believed to be responsible for this phenomenon, i.e. the main carrier determinants are presumably located in this molecular region [4]. However the immunological importance of other sites of the insulin molecule has also been reported [5-7].

Contrary to earlier statements [8], Kobayashi et al. [9] attributed a reduced antigenic activity to Leu-B30 insulin as well as to other B30 analogues, even though the original biological activity is maintained. The present investigation was designed to study the dependency of substitutions in position B30 on both the antigenicity and immunogenicity of the insulin molecule.

\section{Materials and methods}

Besides the original insulins, two analogues of beef insulin and six analogues of pork insulin were at our disposal. In the preparations, the $\mathrm{B} 30$ position was substituted with either threonine, phenylalanine, valine, leucine, glycine or tyrosine; in one case des-alanine B30 was employed. All insulin preparations were crystallized and thereafter chro- matographed on a molecular sieve. In addition, another purification step in the form of ion exchange followed. To map out the biological properties of these novel substances, both the blood glucose lowering effect and the immunological behaviour were assessed and the data obtained compared with those of natural insulin preparations.

Domestic rabbits with an average weight of $2.2 \mathrm{~kg}$ and young adult beagle dogs were used for determining the blood glucose lowering activity. Following the subcutaneous administration of the respective insulin preparation, blood samples were collected at periodic intervals and, subsequently, glucose values were measured by means of the hexokinase method using a commercial kit (Boehringer, Mannheim). The immunological characteristics of the analogues were investigated by using two different approaches. To study the immunogenic behaviour, three different species (pigs, rabbits and dogs) served as experimental animals. Following the initial administration of $1 \mathrm{IU} / \mathrm{kg}$ body weight, weekly booster doses of a logarithmically reduced amount of the preparation was given subcutaneously. Starting from week 6 until the end of the 13-week trial, the uniform doses amounted to $0.2 \mathrm{IU} /$ $\mathrm{kg}$. The initial and following four booster doses were mixed with equal volumes of complete Freund's adjuvant immediately prior to the injections. Accordingly, control groups of experimental animals received the respective original insulin at the same time and in the same dosage.

The antigenicity of the insulin preparations was also evaluated in a model in vitro. Varying amounts of the analogues were added to an established system of labelled pork insulin and preformed anti-insulin antibodies. The degree of inhibition of tracer binding was used as parameter of antigenicity of the respective analogue. 


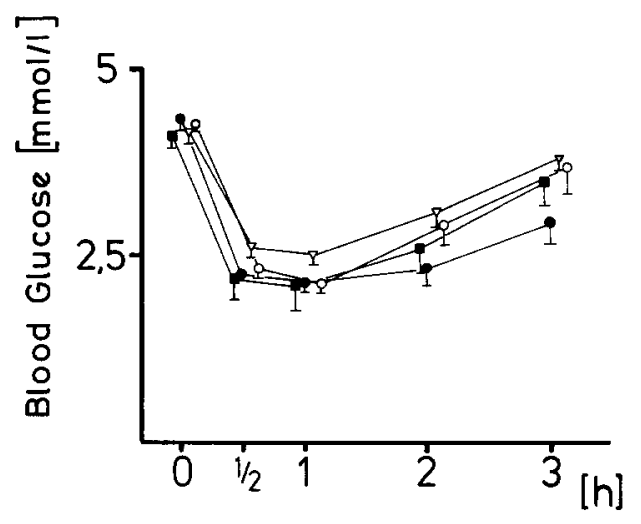

Fig. 1. Blood glucose concentrations in rabbits after injection of original and modified pork insulins. Dissolved, chromatographed pork insulin $(-0)$, porcine Leu-B30 insulin $(\mathrm{O}-\mathrm{O})$, porcine Tyr-B30 insulin $(\square-\square)$ and porcine Phe-B30 insulin $(\nabla-\nabla)$ were administered subcutaneously in doses of $0.2 \mathrm{IU} / \mathrm{kg}$ body weight. Each experimental group consisted of five animals and the results are expressed as mean \pm SEM

\section{Statistical analyses}

Immunogenicity and blood glucose lowering activities are reported as mean values \pm SD or SEM. Statistical significance was calculated by two-tailed Student's t-test.

\section{Results}

The blood glucose-lowering efficacy of the analogues is reflected in one representative graph (Fig. 1). In rabbits both the original and the modified insulins caused a decrease of the blood glucose levels of approximately 2.0-2.5 mmol/1 within $30 \mathrm{~min}$ after subcutaneous administration. Not only the other porcine B30 analogues but the preparations of bovine origin also acted in a similar manner. As expected, the extent of the blood glucose reduction was not as pronounced in dogs, but again no significant difference between the natural hormones and the respective analogue could be interpreted.

Studies of the immunogenic behaviour revealed similar results, i.e. the individual analogues stimulated antibody development to the same extent as did the respective original hormone. Beef insulin and its two analogues proved to be highly immunogenic, while pork insulin and the seven B30 derivatives thereof were not able to provoke antibody production to any considerable extent - at least not within the 12 weeks of the trial. The bovine Thr-B30 insulin was just as immunogenic in rabbits and dogs as it was in pigs.

The investigations concerning the antigenic properties of B30-substituted insulin analogues yielded somewhat different results. In our in vitro system, the degree of competitive binding of the preparations ranged between 73 and 100\% (Fig. 2).

The original pork insulin was set at $100 \%$, since the assay system is based on porcine insulin. The values obtained proved that $\mathrm{B} 30$ derivatives of the porcine hormones were less antigenic than the original porcine hormone itself. In our assay system, this reduction ranged between $10 \%$ and $20 \%$. At first sight, the binding quotas of beef insulin and its analogues to our antibody system surprised because of the low values measured. Beef insulin exhibited only $80 \%$ binding capacity, whereas the binding of the B30-Leu analogue showed an even further reduction to $73 \%$. Interestingly, the binding capacity of the B30-Thr compound was more comparable to pork insulin and its analogues than it was to the beef insulin preparations.
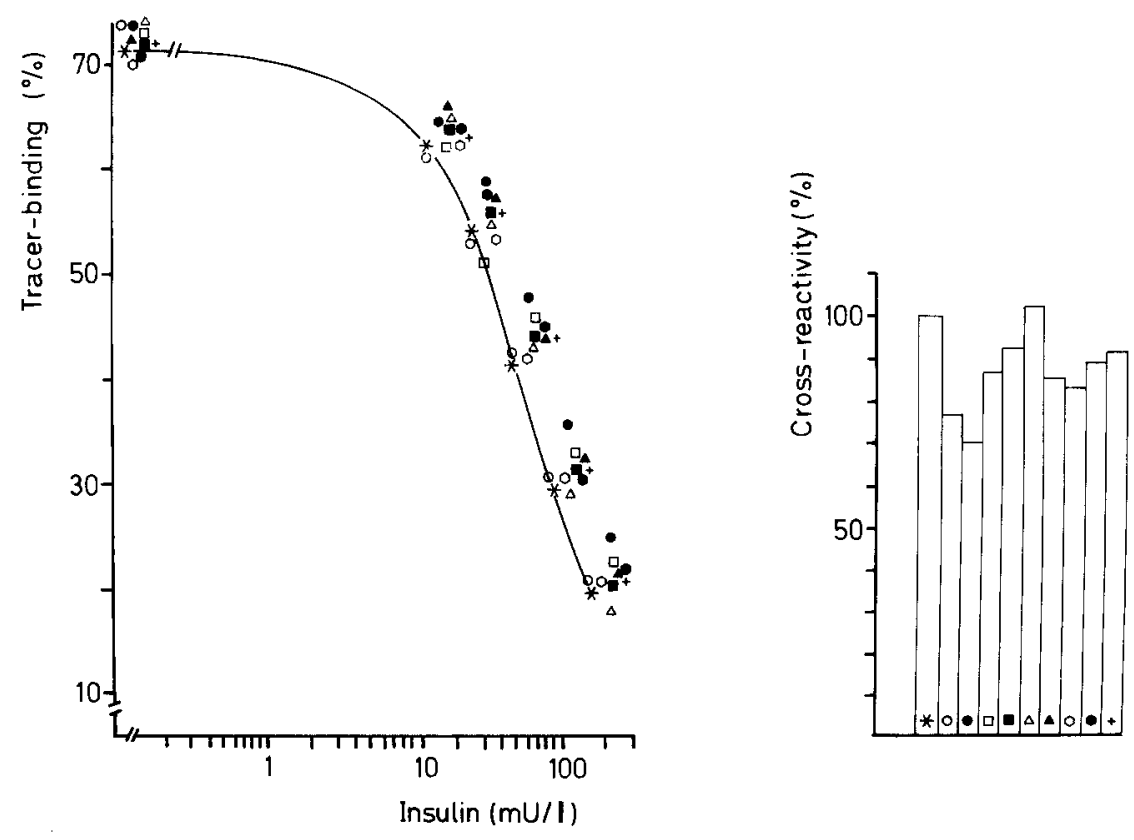

Fig. 2. Antigenicity of insulin B30 derivatives expressed by their competitive binding to preformed anti-pork-insulin antibodies (guinea pig origin). Symbols indicate the insulin or analogue concerned: porcine insulin $*$; bovine insulin- $O$; Leu-B30 insulin ${ }^{(b)}$; Thr-B30 insulin ${ }^{(b)} \square$; Des-Ala-B30 insulin $^{(p)}$; human insulin (semi-synthetic) $^{(\mathrm{p})} \Delta ;$ Tyr-B30 insulin ${ }^{(\mathrm{p})} \mathbf{\Delta}$; Phe-B30 insulin ${ }^{(\mathrm{p})} \circ$; Leu-B30 insulin ${ }^{(\mathrm{p})} \bullet$; Gly-B30 insulin ${ }^{(\mathrm{p})}+$. Assay system: anti-p-insulin- $A B / p$-insulin tracer PEG. ${ }^{(p)}=$ porcine origin, ${ }^{(b)}=$ bovine origin 


\section{Discussion}

The observation that the antigenicity of certain insulin analogues is diminished agrees with the results of earlier studies reported by different groups. Kerp et al. [5] stated that, in comparison with bovine insulin, the binding capacities of high affinity antibodies against desPhe-B1 insulin or des-(Phe-Val)B 1-2 insulin are reduced by $77 \%$ and $76.3 \%$ respectively, with a further decrease to $68 \%$ for des-(phe-Val-Asn)B1-3 [Pyr-B4] insulin. Similar results with other analogues were published by Geiger et al. [6] and later by Kobayashi et al. [9]. All these studies come to the conclusion that certain changes in the amino-acid sequence may influence antigenic behaviour. With regard to the immunogenic properties of insulin preparations, there is general agreement that species-specificity plays a major part in antibody development. Our findings are in accordance with these earlier reports.

However, simultaneous studies of immunogenicity and antigenicity of the individual analogues prompted us to compare the two properties with each other. It appeared of interest to learn whether or not a correlation between the degree of binding to pre-formed antibodies and stimulation of antibody development does exist. A reduction of binding affinity to pre-formed antibodies does not justify the conclusion that a substance must also be less active with regard to antibody stimulation and vice versa.

\section{References}

1. Renold AE, Soeldner JS, Steinke J (1964) Immunological studies of homologous and heterologous pancreatic insulin in the cow. Ciba Found Colloq. Endocrinology 15: 122-138

2. Chance RE, Roof MA, Galloway JA (1976) The immunogenicity of insulin-preparations. Acta Endocrinol 83 (Suppl 205) 185-98

3. Neubauer HP, Schöne H-H (1978) The immunogenicity of different insulins in several animal species. Diabetes 27:8-15

4. Keck K (1975) Ir-gene control of immunogenicity of insulin and Achain loop as a carrier determinant. Nature 254:78-79

5. Kerp L, Steinhilber S, Kasemir H, Han J, Henrichs HR, Geiger R (1974) Changes in immunospecificity and biologic activity of bovine insulin due to subsequent removal of the amino acid $B_{1}, B_{2}, B_{3}$. Diabetes 23: 651-656

6. Geiger R, Obermeier R, Teetz V, Uhmann R, Summ HD, Neubauer H, Geisen K, Regitz G (1980) Biological activity of insulin analogues substituted at the amino group of $\mathrm{B}_{1}$-phenylalanine. In: Brandenburg D, Wollmer A (eds) Chemistry structure and function of insulin and related hormones, Walter de Gruyter, Berlin, New York pp 409-415

7. Neubauer HP, Schöne H-H (1980) Studies on the immunological properties of natural and modified insulins. In: Brandenburg D, Wollmer A (eds) Chemistry, structure and function of insulin and related hormones. Walter de Gruyter, Berlin, New York, pp 578-583

8. Berson SA, Yalow RS (1963) Antigens in insulin determinants of specificity of porcine insulin in man. Science 139: 844-845

9. Kobayashi M, Ohgaku S, Iwasaki M, Shigeta Y, Oka T, Morihara $\mathrm{K}$ (1981) Immunoreactivity and biologic activity of semisynthetic Leu-B-30-insulin. Diabetes 30: 519-522

\section{Dr. H.P. Neubauer}

Pharmaceutical Research Division

Hoechst AG

D-6000 Frankfurt am Main 80

FRG 